Document downloaded from:

http://hdl.handle.net/10251/66158

This paper must be cited as:

Amarajothi, DM.; Alvaro Rodríguez, MM.; García Gómez, H. (2011). Metal-organic frameworks as heterogeneous catalysts for oxidation reactions. Catalysis Science and Technology. 1(6):856-867. doi:10.1039/c1cy00068c.

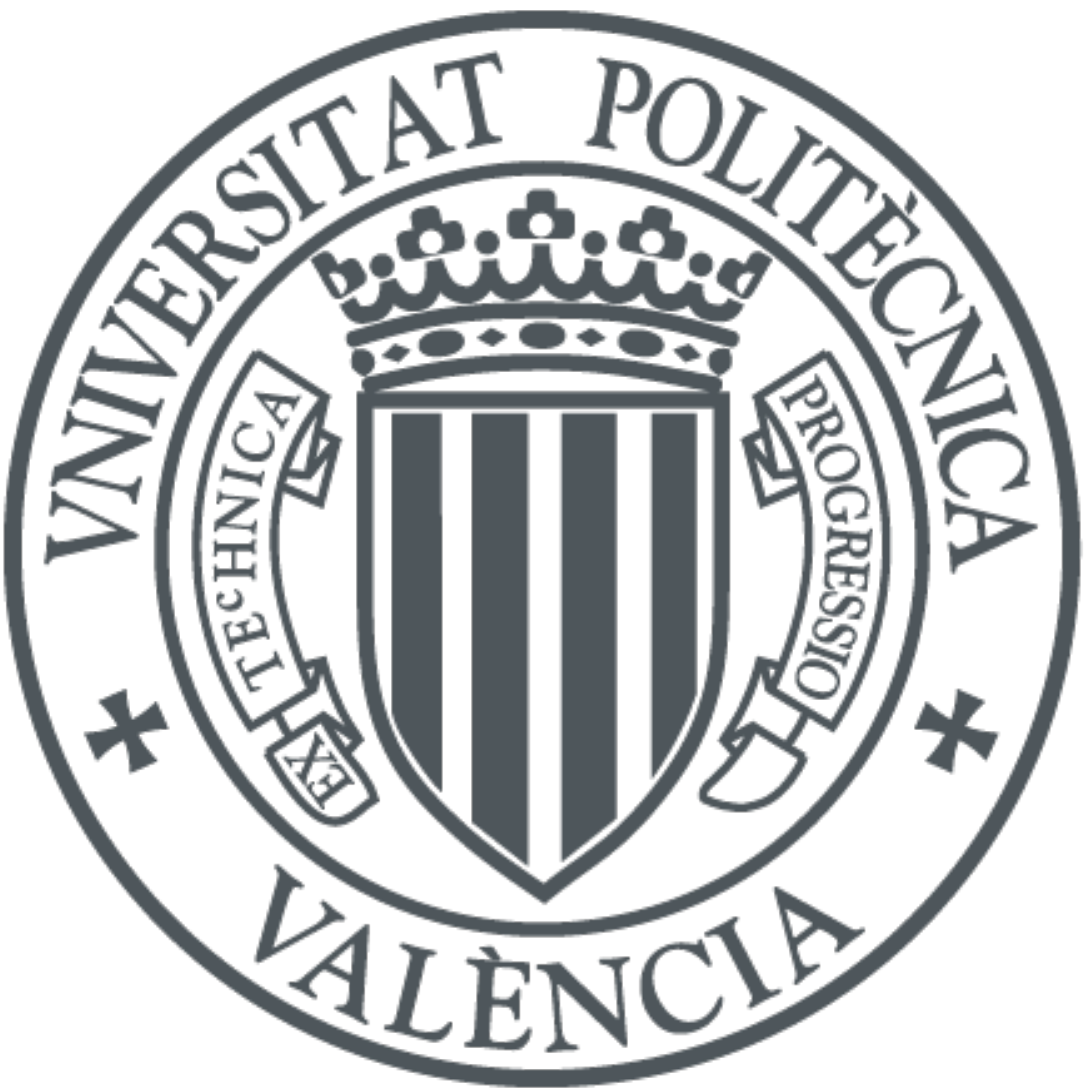

The final publication is available at

http://dx.doi.org/10.1039/c1cy00068c

Copyright Royal Society of Chemistry

Additional Information 


\title{
Metal Organic Frameworks as Heterogeneous Catalysts for Oxidation Reactions
}

\author{
Amarajothi Dhakshinamoorthy, Mercedes Alvaro and Hermenegildo Garcia* \\ Instituto Universitario de Tecnología Química CSIC-UPV and Departamento de Química, \\ Universidad Politécnica de Valencia, Av. De los Naranjos s/n, 46022 Valencia, Spain. \\ Fax: (+) 3496387807 \\ E-mail: hgarcia@qim.upv.es
}

\begin{abstract}
In this Perspective, we describe the use of metal organic frameworks (MOFs) as heterogeneous catalysts for oxidations using hydroperoxides or molecular oxygen. These two types of oxidants fulfill the requirements of green chemistry in terms of environmental benignity and sustainability. For the sake of clarity and to illustrate the possibilities of MOFs as oxidation catalysts, we have constrained ourselves to present the results obtained for the oxidation of cycloalkanes, oxidation of benzylic positions, oxidation of cycloalkenes and aerobic oxidation of alcohols. The use of MOFs as catalysts for enantioselective oxidations has been dealt with in a separate section in which the peculiarity of the required oxidants and the advantages of having homo chiral MOFs have been discussed. The key features of MOFs as catalysts, the similarities with inorganic porous solids and future developments in this field have been discussed in separate sections.
\end{abstract}




\section{Introduction}

Metal organic frameworks (MOFs) are crystalline porous materials whose structure is defined by metal ions or clusters of metals that are connected to a bi or tripodal rigid organic linkers. ${ }^{1-4}$ The directionality of metal ligand interaction and the angles defined by the multipodal linker determine the formation of cavities and channels of nanometric dimensions that can be accessed from the exterior allowing mass transfer and the incorporation of substrates inside the crystal. ${ }^{3}$ MOFs are characterized by being at the top of the list in the porous materials with the largest surface area, lowest framework density and higher pore volume. ${ }^{5}$ As an example, Figure 1 shows a general MOF structure.

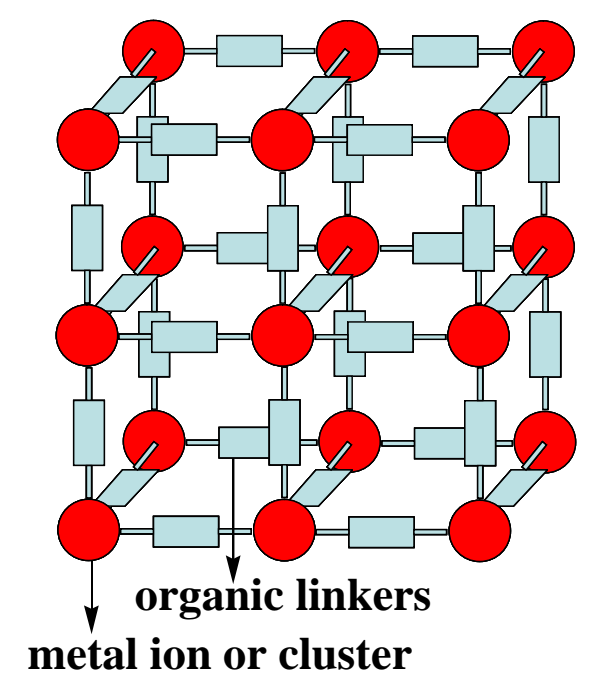

Figure 1. General structure of MOFs.

One additional characteristic of MOFs is that sometimes it is possible to predict the crystal structure of new materials based on the knowledge of metal clusters, their preferred directionalization and geometry and dimensions of the organic linkers. ${ }^{6,7}$ To illustrate this point Figure 2 shows how a series of aromatic dicarboxylates that can be used as linkers for the synthesis of isomorphic MOFs with identical crystal structure but increasing dimensions of unit cell. 
<smiles>O=C([O-])c1ccc(C(=O)[O-])cc1</smiles><smiles>COC(=O)c1ccc2cc(C(=O)OC)ccc2c1</smiles><smiles>O=C([O-])c1cc2c3c(c1)CCc1cc(C(=O)[O-])cc(c1-3)CC2</smiles>

NDC<smiles>Cc1c(C)c(C(=O)[O-])c(C)c(C)c1C(=O)[O-]</smiles>

TMBDC<smiles>COC(=O)c1ccc(-c2cc(-c3ccc(C(=O)[O-])cc3)cc(-c3ccc(C(=O)[O-])cc3)c2)cc1</smiles>

BTB

\section{IRMOF-1 IRMOF-8 IRMOF-11 IRMOF-18}

MOF-177

$A_{\text {surf }} 3362 \mathrm{~m}^{2} / \mathrm{g} \quad A_{\text {surf }} 1466 \mathrm{~m}^{2} / \mathrm{g} A_{\text {surf }} 1911 \mathrm{~m}^{2} / \mathrm{g} A_{\text {surf }} 1501 \mathrm{~m}^{2} / \mathrm{g}$

$A_{\text {surf }} 4526 \mathrm{~m}^{2} / \mathrm{g}$

Figure 2. Aromatic dicarboxylates varying in length used as linkers for a series of isoreticular MOFs. The common acronym of the linker and MOF as well as the resulting surface area have also been indicated.

Beyond the synthesis of new materials, the interest in MOFs derives from the large porosity and its potential use as adsorbents for hydrogen, carbon dioxide and other gases. $^{8-14}$ It has also been reported that MOFs can be used for the separation and sequestration of some molecules present in gas phase. ${ }^{15}$ Scheme 1 shows the expected possible applications of MOFs in different sectors.

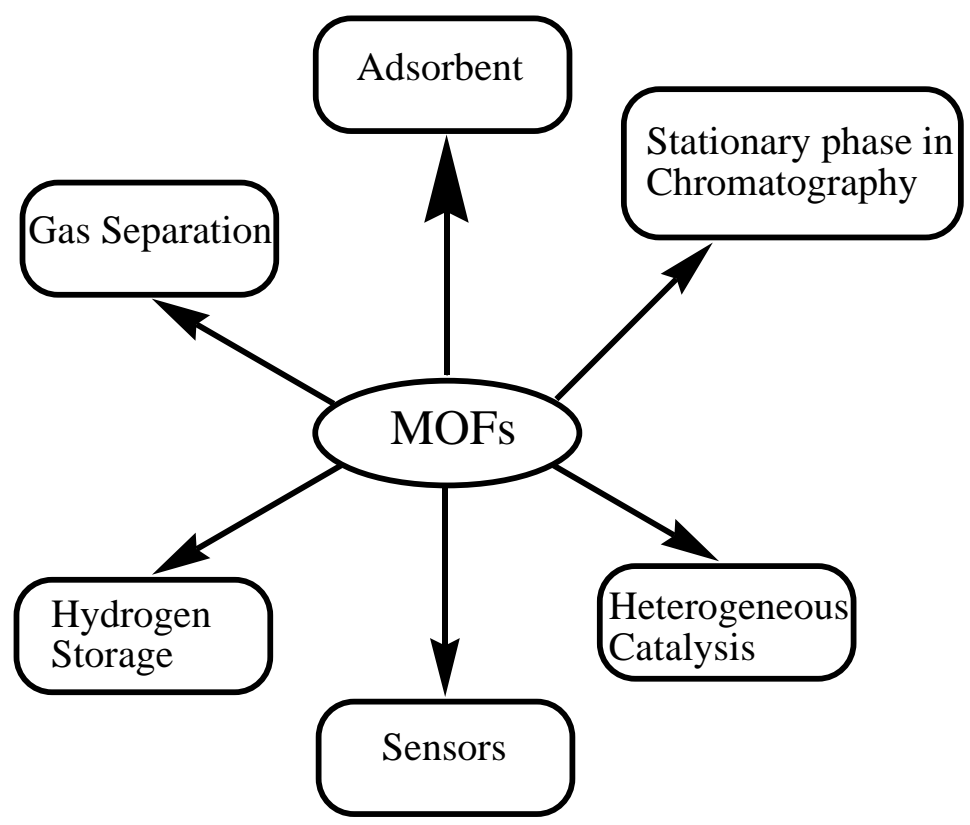


Scheme 1. Possible applications of MOFs in various areas

\section{MOFs versus zeolites}

MOFs have crystal structures that are related to those of zeolites and other inorganic porous solids. Considering the high metal content in MOFs and its similarity with zeolites, these materials are attracting an increasing interest as heterogeneous catalysts. ${ }^{16-28}$ Table 1 provides a comparison of some features that are relevant in catalysis for zeolites and MOFs.

Table 1. Comparison of MOFs with zeolites with some properties relevant to catalysis

\begin{tabular}{|c|c|c|}
\hline Properties & Zeolites & MOFs \\
\hline Lewis acidity & high & Low to medium \\
\hline Brønsted acidity & $\begin{array}{l}\text { Bridging } \\
\text { groups }\end{array}$ & $\begin{array}{l}\text { Introduced by post-synthetic } \\
\text { modifications or functional } \\
\text { present in the ligands not } \\
\text { compromised in the structure }\end{array}$ \\
\hline Surface area & Around $200-500 \mathrm{~m}^{2} / \mathrm{g}$ & $\mathrm{Up}$ to $5000 \mathrm{~m}^{2} / \mathrm{g}$ \\
\hline Pore volume & Around $0.1-0.5 \mathrm{~cm}^{3} / \mathrm{g}$ & over $1 \mathrm{~cm}^{3} / \mathrm{g}$ \\
\hline Thermal stability & $>450{ }^{\circ} \mathrm{C}$ & $300{ }^{\circ} \mathrm{C}$ \\
\hline $\begin{array}{l}\text { Chemical } \\
\text { stability }\end{array}$ & high & $\begin{array}{l}\text { limited and incompatible with } \\
\text { certain solvents }\end{array}$ \\
\hline Diffusion & $\begin{array}{l}\text { High for small molecules in the } \\
\text { gas phase and slow in the liquid } \\
\text { phase }\end{array}$ & $\begin{array}{l}\text { Strongly influenced by the } \\
\text { polarity of linkers }\end{array}$ \\
\hline Basicity & $\begin{array}{l}\text { Arises from the framework } \\
\text { oxygens }\end{array}$ & $\begin{array}{ll}\text { introduced by } & \text { post-synthetic } \\
\text { modifications } & \text { or directly } \\
\text { through linkers } & \end{array}$ \\
\hline $\begin{array}{l}\text { Metal site } \\
\text { density }\end{array}$ & low & High \\
\hline $\begin{array}{l}\text { Framework } \\
\text { defects }\end{array}$ & $\begin{array}{l}\text { Plays an important role in many } \\
\text { reactions }\end{array}$ & Expected to play a minor role. \\
\hline $\begin{array}{l}\text { Active site } \\
\text { environment }\end{array}$ & $\begin{array}{l}\text { Mostly hydrophilic but can also be } \\
\text { made hydrophobic }\end{array}$ & Considerably hydrophobic \\
\hline $\begin{array}{l}\text { Poisoning of } \\
\text { active sites }\end{array}$ & Reactivation by thermal treatment & $\begin{array}{l}\text { Thermal treatment not valid for } \\
\text { catalyst regeneration }\end{array}$ \\
\hline
\end{tabular}




\begin{tabular}{lll}
\hline Chirality & Not possible or difficult to achieve & $\begin{array}{l}\text { Homochiral solids can be easily } \\
\text { obtained from chiral linkers or } \\
\text { postsynthetic modifications }\end{array}$ \\
\hline
\end{tabular}

As it can be seen there, according to our view, zeolites are much more suited as solid catalysts for gas phase reactions but due to its limited available pore size they generally undergo fast deactivation for liquid phase reactions. In addition most of the efforts to develop zeolites with large pore size in the nanometer scale have met with failure. $^{29,}{ }^{30}$ In liquid phase reactions, diffusion can be a controlling process and also considering the larger molecular dimensions of substrates that can be employed in liquid phase process, it would be highly important to have porous catalysts with pore dimensions larger than those of classical zeolites.

In this regard, MOFs can be considered as complimenting an expanding the work of zeolites in heterogeneous catalysis. Certainly, due to the low thermal stability of MOFs are not likely to be the potential interest for gas phase reactions. On the contrary the large versatility in the design and engineering of MOFs with adequate geometry and pore size make these materials more promising for liquid phase reactions, just the type of transformations for which the performance of zeolites is generally not adequate. Although there are some scattered examples for which zeolites are excellent solid catalysts in the liquid phase such as selective hydrogenation of trans-fatty acids on Pt-ZSM-5 zeolite ${ }^{31}$ or selective macrolactonation hydroxycarboxylic acids on HY zeolites, $^{32}$ there are many other cases in where zeolites become immediately deactivated due to poisoning when the reaction is carried out in the liquid phase.

Finally, one field in which MOFs are certainly much better materials than zeolites is to promote enantioselective reactions. Using chiral linkers, preparation of homochiral MOFs can be easily obtained and these solids could be suitable as catalysts for asymmetric reactions. ${ }^{33}$ Besides hydrogenations, asymmetric oxidations is the field 
that has attracted considerable attention since the resulting products and, particularly chiral epoxides, are very important synthetic intermediates in asymmetric reactions. ${ }^{34}$

\section{Stability of MOFs}

In the present perspective, we are going to comment on the use of MOFs as catalysts for liquid-phase oxidations. As in any use of MOFs in catalysis, the key points to be addressed are their activity, selectivity, stability under the reaction conditions and deactivation pathways. Thus, in addition of providing conversion and selectivity data, when using MOFs it is also necessary to determine the stability of the material and the lack of leaching of active metal species to the solution. These points are typically addressed by checking the crystal structure and determining the surface area after the catalytic reactions as well as performing analysis of the solutions at final reaction time. Productivity, defined as the amount of product that can be obtained with a given amount of catalyst before deactivation, is also one important data that must be considered in order to assess the feasibility of using MOFs as catalysts.

Concerning the use of MOFs as oxidation catalyst, there is a considerable interest in green chemistry to replace stoichiometric oxidations using transition metals or other environmentally unfriendly oxidants by environmentally benign ones. ${ }^{20}$ The ultimate green oxidants in terms of the percentage of active oxygen and toxicity of the byproducts are hydrogen peroxide, organic hydroperoxides and oxygen. ${ }^{35}$ Oxidation using peroxides and oxygen require the use of suitable catalyst and those based on transition metals are of general use. While metal oxides and another inorganic compounds are non porous, the catalytic activity per metal atom (turnover number, TON) tend to be low and in order to increse these TON values a reduction of particle size down to the nanometer scale is necessary. Evenfor nanoparticulate materials, it would be convenient to have all the metal sites exposed on the surface of the solid. This 
strategy to isolate transition metal atoms on a large surface area solid has been developed by having transition metal containing zeolites and using them as oxidation catalysts. $^{36}$ The activity of these metal containing zeolites for many oxidations particularly in the gas phase has been found to be very high and they have been used in several industrial processess. ${ }^{37,} 38$ However, one disadvantage of transition metal containing zeolites is the low metal content that can be achieved. This limitation can be overcome using MOFs as catalysts. Since these solids can have weight percentage of transition metals over $20 \%$ (typically zeolites contains only a few percentage of transition metals) and these metals are dispersed in a very large surface area.

One point to be addressed in any heterogeneous catalysts, but also of particular relevance in MOFs, is the stability of the catalyst and in particular the absence of metal leaching. For instance in the case of transition metal containing zeolites metal leaching under optimum conditions in the presence of oxidizing agents is a general problem that has to be carefully surveyed and in the case that the total absence of leaching is proposed it has to be convincingly proved. A strong oxidant generates stress in the catalytic sites since the mechanism can require either reversible changes in the oxidation state or a cycle of ligand exchange. Therefore going from one step to the other in the mechanism typically requires changes in the coordination sphere in the metal ion and these changes can be large enough to effect the migration of the metal out of the solid framework. In the case of MOFs, one additional problem is the stability of the organic linker to the oxidative stress and the strength of the metal-cluster linker interaction that could be not strong enough to completely avoid metal leaching under the typical conditions and solvents used in oxidation reactions.

\section{Suitable oxidizing reagents}


One of the principles of Green Chemistry is the replacement of stoichiometric by catalytic reactions. ${ }^{39}$ Oxidation reaction is a typical case in which for environmental reasons the change of stoichiometric oxidants for catalytic process is particularly important due to the toxicity of the resulting waste. Specially, oxidizing reagents containing transition metals such as $\mathrm{KMnO}_{4}, \mathrm{~K}_{2} \mathrm{Cr}_{2} \mathrm{O}_{7}, \mathrm{~Pb}(\mathrm{OAc})_{4}$ and other should be avoided. ${ }^{40}$ Among the oxidizing reagents that are environmetally benign, the most important ones are hydrogen peroxide, organic hydroperoxides and molecular oxygen. In the present Perspective and for the sake of conciseness, we will focuss on these reports that have described the use of MOFs as oxidation catalysts for these three types of oxidants considered "green". While hydrogen peroxide is almost perfect as oxidant due to high oxygen content and the formation of water as the only byproduct, the two main problems of this reagent is the lack of miscibility in organic solvents and its high cost. Organic hydroperoxides have the advantage of being soluble in most organic solvents and miscible with most organic substrates, but again the cost of these reagents is high.

When employing hydroperoxides one point that should always be studied is the selectivity of the process with respect to these reagents. In other words, besides selectivity with respect to organic substrates, it is also necessary to know which percentage of hydroperoxides have been decomposed spureously by the catalyst and which percentage have been successfully employed to transform the organic substrates, In general oxidant to substrates molar ratios as close as possible to the unity should be employed. Even more, frequently the substrate is cheaper than the hydroperoxides and, then, the oxidizing reagent can be employed in substoichiometric amounts to maximize its selectivity towards oxidation. 
In terms of cost and availability, molecular oxygen is the ideal terminal oxidant but the challenge is how to direct its reactivity towards a single product. Oxidations using molecular oxygen are generally characterized by a considerable lack of selectivity and the formation of complex reaction mixtures that are frequently useless from the industrial point of view. One way to circumvent this lack of selectivity when using oxygen is to keep conversion low while in the case of use of hydroperoxides conversion and selectivity can be much higher and this can justify their use.

One special comment deserves the reagents employed in asymmetric synthesis. In this case, transmission of chirality to the products typically requires a bulk reagent that increases the free energy discrimination between the two transition states leading to each enantiomer. For this reason, N-oxides, iodosylbenzene are nucleophilic cocatalysts are typically employed to enhance asymmetric induction. ${ }^{41}$

This Perspective has been organized by presenting examples of the use of MOFs as oxidation catalysts for a few organic compounds such as cycloalkanes and alkenes and alcohol oxidation. A final section deals with the use of MOFs as solid catalyst for enantioselective oxidations. The reader should consult the recent reviews covering the use of MOFs as catalysts for organic reactions for a comprehensive coverage of the use of MOFs as heterogeneous catalysts. ${ }^{1,}{ }^{16}$ Rather than being exhaustive, our purpose has been to describe the cutting edge of the current status of MOFs as oxidation catalysts using hydroperoxides/oxygen. We have constrained ourselves to examples that can be of large industrial or synthetic relevance such as oxidation of hydrocarbons and alcohols.

\section{Oxidation of cycloalkanes}

A copper-based MOFs having a trinuclear triangle cluster namely $\left[\mathrm{Cu}_{3}\left(\mu_{3}-\mathrm{OH}\right)(\right.$ $\left.\mu-\mathrm{pz})_{3}(\mathrm{EtCOO})_{2}\left(\mathrm{H}_{2} \mathrm{O}\right)\right](\mathrm{Hpz}=$ pyrazole $)$ has been reported for peroxidative oxidation of 
cyclopentane and cyclohexane using hydrogen peroxide as oxidant in combination with nitric acid in acetonitrile/water medium under mild conditions. ${ }^{42}$ It has been shown that the presence of nitric acid is essential for the occurrence of the oxidation reaction in very high overall selectivity (presumably close to $100 \%$ ) toward the formation of the corresponding alcohols and ketones. In all the conditions tested, cyclohexanol $(25.1 \%)$ was the major product accompained with a small amount of cyclohexanone $(2.8 \%)$. No traces of typical byproducts for peroxidative oxidations of cycloalkanes namely diketones, diols, etc were observed. Increasing the amount of catalyst and hydrogen peroxide resulted in incresing the percentage conversion. It is, however, remarkable that this copper MOF could be stable in the presence of nitric acid and water. Since as commented earler, MOF stability is a key issue in heterogeneous catalysis convincing evidence of long-term stability should be presented.

Recently, iron coordinated to 1,3,5-benzenetricarboxylate (BTC) [Fe(BTC)] metal organic framework has been commercialized by BASF and is available from Sigma Aldrich under the name of Basolite F 300. Although, the exact crystal structure of $\mathrm{Fe}(\mathrm{BTC})$ is still unknown, it is likely to possess a similar structure and environment as in MIL-100 ${ }^{43}$ (Fe(III) coordinated with 1,3,5-benzenetricarboxylate), a highly crystalline MOF whose structure has been solved, but exhibits a different XRD than the commercial Basolite F 300. The aerobic oxidation of cyclooctane has been reported using N-hydroxyphthalimide loaded on $\mathrm{Fe}(\mathrm{BTC})$ [NHPI/Fe(BTC)] as heterogeneous catalyst under solvent free conditions (Scheme 2). ${ }^{44}$ It has been demonstrated that a selectivity to cyclooctanol and cyclooctanone above $90 \%$ could be achieved at $30 \%$ conversion in $6 \mathrm{~h}$. Increasing the reaction time further, decreases the selectivity towards cyclooctanol and cyclooctanone, a fact that is general in those reactions in which the primary reaction products can undergo secondary reactions. On the other hand, aerobic 
oxidation of tetralin showed the selectivity of tetranol and tetralone to $90 \%$ in $3 \mathrm{~h}$ with $33 \%$ conversion. This observed selectivity is quite comparable to other catalytic system reported with peroxides as oxidant and supports the operation of a radical chain mechanism for the autooxidation reaction.

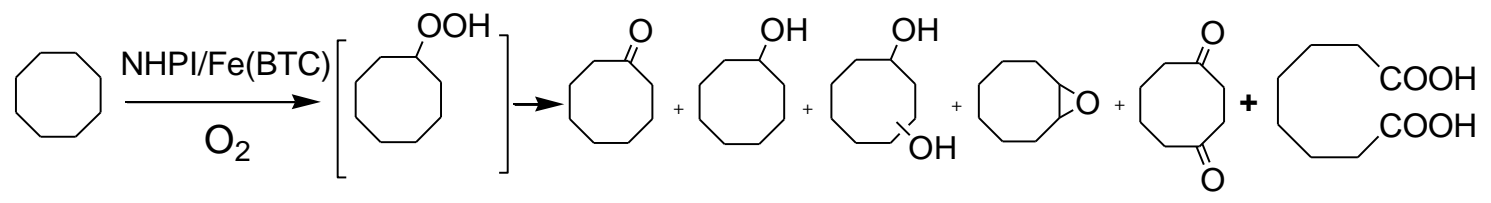

Scheme 2. Aerobic oxidation of cyclooctane catalyzed by NHPI/Fe(BTC).

The progress in this area should be done by showing the generality of this NHPI/Fe(BTC) system for the aerobic oxidation of other hydrocarbons including the industrially relevant cyclohexane and also acyclic alkanes. What appears reasonable is that considering that autooxidation using air is a general process, the target should be a better control of the selectivity at reasonable conversions. MOF as catalysts can be suitable materials to achieve this goal since confinement of radicals in a restricted reaction cavity can be a general strategy to control their reactivity. It is interesting to note that due to the diffusion limitations conventional large pore zeolites and particularly $\mathrm{NaY}$ was found to be not adequate to develop analogous NHPI/Fe-NaY system. Also, Jacobs and co-workers reported an NHPI loaded on silica and observed that the activity and selectivity towards cyclohexane oxidation was low. ${ }^{45}$

\section{Oxidation of benzylic compounds}

Aerobic oxidation of alkyl aromatics and benzylic positions is a process of large industrial relevance that can be typically promoted by metal carboxylates in strong acid media. $^{46}$ Since, most MOFs are crystalline porous metal carboxylates there is a reasonable chance that they can also be active for this reaction, although the problem of stability against strong acids is going to pose a strong limitation to the use of MOFs. 
Due to the boiling point and easy analysis of the reaction products, tetralin (see structure) can be considered a suitable model compound for this important oxidation.<smiles>c1ccc2c(c1)CCCC2</smiles>

Figure 3. Structure of tetralin.

MIL-101 (chromium terephthalate) has been used as catalyst for oxidation of tetralin in the presence of $t$-butyl hydroperoxide (TBHP) as well as pivalaldehyde/ $\mathrm{O}_{2}$ as oxidants. ${ }^{47}$ Under optimized conditions, oxidation of tetralin resulted in $70 \%$ conversion with $84.8 \%$ selectivity with TBHP (3 eq), while in the presence of pivalaldehyde/ $\mathrm{O}_{2}$ MIL-101 promotes higher tetralin conversion and selectivity reaching $93 \%$ and $86 \%$, respectively. The nature of solvent and the removal of water were found to play a vital role. The catalyst showed very good stability under both reaction conditions and was reused for five runs without much loss in the catalytic activity using TBHP as oxidant. ${ }^{47}$

We were interested in exploring the catalytic activity of commercially available $\mathrm{Fe}(\mathrm{BTC})$ as oxidation catalyst and hence oxidation of xanthene was tested with oxygen and hydrogen peroxide and resulted in no oxidation. In contrast, the combination of $\mathrm{Fe}(\mathrm{BTC})$ as catalyst and TBHP as oxidant leads to the transformation of xanthene to xanthone (Scheme 3). ${ }^{48}$ Under the optimized reaction conditions, more than $90 \%$ yield of xanthone was achieved with 1.5 equivalent of TBHP. Similarly, $\mathrm{Cu}_{3}(\mathrm{BTC})_{2}$, a MOF that is also supplied by Sigma Aldrich as Basolite C 300, showed a slightly lower catalytic activity than Fe(BTC). ${ }^{48}$ The scope of these commercial MOFs was expanded to the oxidation of other benzylic compounds with high selectivity.

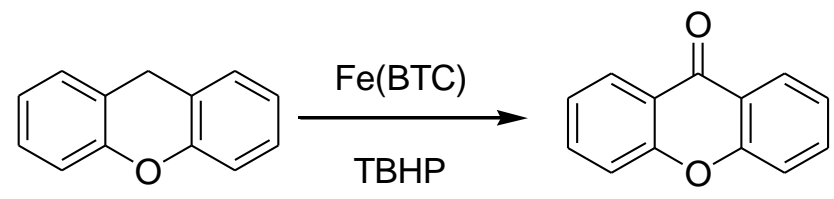


Scheme 3. Oxidation of xanthene to xanthone catalyzed by Fe(BTC) using TBHP.

Aerobic oxidation of tetralin has been reported with $\mathrm{Cu}(2-\text { pymo })_{2}(2-$ pymo $=2$ hydroxypyrimidinolate $)$ and $\mathrm{Co}(\mathrm{PhIM})_{2}(\mathrm{PhIM}=$ phenylimidazolate $)$ based $\mathrm{MOF}$ as heterogeneous catalysts. ${ }^{49}$ When the aerobic oxidation was performed with $\mathrm{Cu}(2$ pymo $)_{2}, 52 \%$ conversion of tetralin was observed accompained by some undecomposed peroxides (Scheme 4). $\mathrm{Co}(\mathrm{PhIM})_{2}$ reached $23 \%$ tetralin conversion under the optimized conditions. The presence of hydroperoxides is highly detrimental for the quality of the reaction mixture of the oxidation of benzylic or cyclic alkanes due to the explosion risk associated with their presence in the reaction medium during the work up. In a way to increase the quality of the autooxidation mixture, minimizing the presence of hydroperoxides, combinations of $\mathrm{Cu}(2-\mathrm{pymo})_{2}$ and $\mathrm{Co}(\mathrm{PhIM})_{2}$ were used in a single catalytic system. It was hoped that in this way, the high yields achieved using $\mathrm{Cu}(2-$ pymo) $)_{2}$ will be accompanied with a low hydroperoxide content due to the ability of $\mathrm{Co}(\mathrm{PhIM})_{2}$ to decompose them. As it was anticipated, the percentage conversion was found to be intermediate between that of a single $\mathrm{Co}(\mathrm{PhIM})_{2}$ and $\mathrm{Cu}(2 \text {-pymo })_{2}$ component accompanied with low amounts of the hydroperoxide. ${ }^{49}$ Both catalysts showed no leaching and could reused without much loss in the catalytic activity.

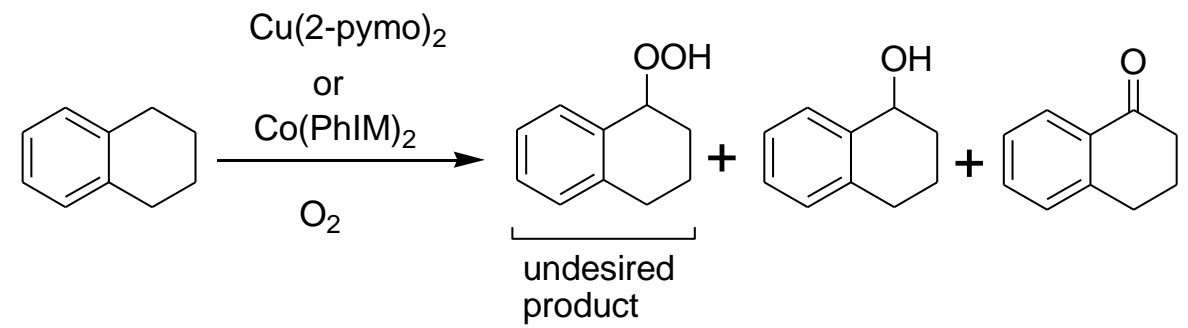

Scheme 4. Aerobic oxidation of tetralin using $\mathrm{Cu}(2-\text { pymo })_{2}$ and $\mathrm{Co}(\mathrm{PhIM})_{2}$

In the previous examples, the constitutional metal ions present on the nodal framework positions are active as catalytic sites due to the presence of exchangable 
coordination positions. But, besides providing active sites, MOFs can also be used as high surface area supports to include metal complexes that will act as the catalytic sites.

In these cases, MOFs act as supports to isolate other active sites incorporated inside the micropores increasing the catalytic activity by increasing dispersion and favoring accessibility. As an example of this strategy, Kegin type of polyoxometallate (POM) namely $\mathrm{SiW}_{12} \mathrm{O}_{40}{ }^{4-}$ has been incorporated in $\mathrm{Cu}_{2}\left(4,4^{\prime}-\text { bpy }\right)_{4}\left(\mathrm{H}_{2} \mathrm{O}\right)_{4}$ (bpy = bipyridine) and used as catalysts for oxidation of ethylbenzene using of TBHP as oxidant.The copper MOF containing POM promoted ethylbenzene oxidation resulted in $56.8 \%$ conversion with $88 \%$ selectivity towards acetophenone at $70{ }^{\circ} \mathrm{C}$, while the homogeneous POM showed only $27.5 \%$ conversion with $76 \%$ selectivity. ${ }^{50}$ The heterogeneous catalyst showed no leaching of copper and could be used three times without loss in the catalytic activity.

In general, when used as homogeneous catalysts for oxidation reactions in the liquid phase metalphthalocyanine (MPc) complexes $^{51}$ exhibit limited reactivity due to aggregation through $\pi$-stacking and the formation of oligomers connected by oxo ligands. Analogously, tetrasulfophthalocyanine $(\mathrm{FePcS})$ was reported as efficient catalyst for the selective oxidation of aromatic compounds with TBHP. ${ }^{52}$ Heterogenization of FePcS over MIL-101 (FePcS/MIL-101) was reported as heterogeneous catalyst for the oxidation of benzyl alcohol using TBHP as oxidant. ${ }^{53}$ The rate of oxidation was decreased when the loading of FePcS was increased from 5 to $15 \%$. This negative effect of excessive guest loading is generally observed and can be attributed to the difficulty to access to the occluded FePcS when the loading is too high and diffusion inside the micropores is severely restricted. Although FePcS/MIL-101 showed better activity than unsupported $\mathrm{FePcS}$, selectivity towards benzaldehyde was lower due to the formation of benzoic acid. However, one of the major problems of 
FePcS/MIL-101 is deactivation. Thus, FePcS/MIL-101 showed $35 \%$ conversion of benzyl alcohol in $15 \mathrm{~min}$, but further increase of the time to $2 \mathrm{~h}$ showed the same conversion. This indicates that the catalyst has undergone deactivation during the course of reaction. This issue is, however, of capital importance in the field of MOF catalysts and efforts should have been devoted to analyze the spent catalyst in order to identify the poisons and the deactivation mechanism. In contrast to the case of benzyl alcohol, oxidation of 2,3,6-trimethylphenol was more facile with FePcS/MIL-101 than with FePcS due to the faster diffusion of aromatic compounds inside the framework. ${ }^{53}$

Hence, it is believed that loading of MPc onto MIL-101 would increase the catalytic activity of this hybrid material in terms of conversion and selectivity by isolating the active site and impeding aggregation and oligomerization. This higher stability of the active sites is reflected in an increase on the TON. For example, FePcF $F_{6} @$ MIL-101 and RuPcF $@$ MIL-101 catalysts showed very high TON of 48200 and 46300 , respectively, for the aerobic oxidation of tetralin to 1-tetralone at $24 \mathrm{~h}$ while the corresponding unsupported $\mathrm{FePcF}_{6}$ showed only 6300. In contrast, $\left(\mathrm{FePc}-t-\mathrm{Bu}_{4}\right)_{2} \mathrm{~N}$ supported on MIL-101 showed similar activity than of $\left(\mathrm{FePc}-t-\mathrm{Bu}_{4}\right)_{2} \mathrm{~N}$ due to the external localization of this large complex on the surface of MIL-101. ${ }^{54}$ This enhancement of catalytic activity of MPc on loading over MIL-101 shows the advantages of supporting and isolating active metal complexes in a large area porous solids.

\section{Oxidation of cycloalkenes}

Oxidation of $\mathrm{C}=\mathrm{C}$ double bonds constitutes important reactions in organic chemistry that can lead to a diverse array of derivatives. One of this type of reactions is epoxidation. Epoxides have a wide range of industrial applications as feedstocks for high added value products. Usually epoxidation is carried out in the presence of an 
oxidant such as hydrogen peroxide, TBHP or peracids. Although hydroperoxides in combination with suitable catalysts constitute an adequate system from the view point of green chemistry, due to their cost it would be desirable to develop alternative systems based on oxygen. Thus, in the present section we will discuss the various possibilities in where MOFs can act as heterogeneous catalysts in combination with one of the above oxidants, with an special emphasis on discussing the aerobic epoxidation. Also, it will be shown how selectivity is altered when the experimental conditions are changed.

$\left[\mathrm{Cu}\left(\mathrm{H}_{2} \text { btec }\right)(\mathrm{bpy})\right]_{\infty}\left(\mathrm{H}_{4} \mathrm{btec}=1,2,4,5\right.$-benzenetetracarboxylic acid and bpy $=2,2^{\prime}-$ bipyridine) has been synthesised and used as catalyst for epoxidation of cyclohexene and oxidation of styrene by TBHP. ${ }^{55}$ Oxidation of cyclohexene at $75^{\circ} \mathrm{C}$ in $24 \mathrm{~h}$ resulted in $64.5 \%$ conversion with 1886 TON and $73.1 \%$ selectivity towards cyclohexene oxide (Scheme 5). This product was also accompanied with $7.7 \%$ and $19.2 \%$ of cyclohexanone and 2-cyclohexenone. On the other hand, oxidation of styrene at $75{ }^{\circ} \mathrm{C}$ resulted in $23.7 \%$ conversion with a TON of 673 in $24 \mathrm{~h}$ with $71 \%$ styrene oxide and $29 \%$ of benzaldehyde. ${ }^{55}$ It is interesting to note that TBHP was used in equimolar amounts and, therefore it appears that the copper MOF exhibits a high selectivity for the oxidation from the point of view of the hydroperoxide consumption, minimizing spureous TBHP decomposition. However, the stability of catalyst in terms of leaching and reusability was not reported and this is an issue of large importance, particularly in oxidation reactions.

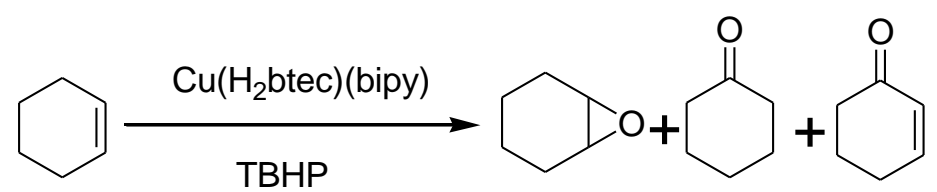

Scheme 5. Oxidation of cyclohexene with TBHP catalyzed by $\left[\mathrm{Cu}\left(\mathrm{H}_{2} \text { btec }\right)(\text { bipy })\right]_{\infty}$.

It is interesting to note that epoxidation using $\mathrm{Ti}$ containing zeolites was a hot topic in heterogeneous catalysis in the 1990 's. ${ }^{56-58}$ The outcome of this research is that 
Ti-Beta zeolite was a suitable solid catalyst for oxidation of alkenes using hydrogen peroxide as reagent, but the selectivity towards epoxide was very low due to generally undesirable, ring opening of epoxides. ${ }^{59}$ In order to increase selectivity towards epoxide absence of water and the use of hydrophobic catalysts with much larger pore sizes was needed. Thus, it appears that silanized Ti-MCM-41 in combination with TBHP is a good catalyst for epoxidation of propene and other alkenes. ${ }^{60}$ The Ti content of the catalysts has to be necessarily low in order to avoid the formation of $\mathrm{TiO}_{2}$ nanoparticles. Also, silanization is necessary to increase the stability of MCM-41 and expel water from the interior of MCM-41. One of the objectives of silanization was to increase the organophilicity and to reduce hydrophilicity of the MCM-41. ${ }^{61}$

In continuous effort to synthesise different MOFs, $\mathrm{Co}_{4}{ }_{4} \mathrm{O}(\mathrm{bdpb})_{3}$ (where $\mathrm{H}_{2} \mathrm{bdpb}$ 1,4-bis[(3,5-dimethyl)pyrazol-4-yl]benzene) was reported and its catalytic activity towards cyclohexene oxidation using TBHP as oxidant studied. ${ }^{62}$ Negligible conversion of cyclohexene was observed in the absence of catalyst, while $27.5 \%$ conversion was achieved in its presence after $22 \mathrm{~h}$. It has to be noted that the highest selectivity was towards the formation of peroxide and lowest towards cyclohexene epoxide (3\%). Further decomposition of peroxide resulted in the formation of cyclohexenone. Powder XRD of the catalyst showed no sign for MOF decomposition and leaching experiments resulted in negligible amount of $\mathrm{Co}(\mathrm{II})$ ions in the hot filteration test. Although a $5 \%$ reduction in the conversion was observed from the first to the second run, further use of catalyst exhibited similar reactivity as the second run. This partial decrease of the catalyst activity was attributed to the pore blockage by organic compounds as evidenced by a decrease in BET surface area from 1485 to $1018 \mathrm{~m}^{2} / \mathrm{g}$. Replacing TBHP with $\mathrm{Ph}_{3} \mathrm{COOH}$ dropped the catalytic activity significantly as the latter bulky hydroperoxide 
could not diffuse into the pores of MOF whereas TBHP is assumed to diffuse freely inside the framework.

Vanadium is one of the transition metals that exhibits general catalytic activity for oxidation reactions. Recently, vanadium containing metal organic framework VMIL-47 has been reported. MIL-47 consists of a porous terephthalate framework built from infinite chains of $\mathrm{V}^{4+} \mathrm{O}_{6}$ octahedra ordered in a three-dimensional orthorhombic structure that exhibits large pores. V-MIL-47 shows catalytic activity towards cyclohexene oxidation in the liquid phase using TBHP (2 eq) (Scheme 6). ${ }^{63}$ The initial TOF exhibited by V-MIL-47 for cyclohexene conversion was $43 \mathrm{~h}^{-1}$ and increasing the time increased the percentage conversion to $50 \%$. In the first $2 \mathrm{~h}$, the formation of cyclohexene oxide reached to $15 \%$ and further time increase leads to the formation $1,2-$ cyclohexanediol decreasing the epoxide selectivity to $5 \%$. On the other hand, a significant amount of $t$-butyl-2-cyclohexenyl-1-peroxide (30\%) was also formed. ${ }^{63}$ It has been observed that the pores of V-MIL-47 were blocked by organic compounds and regeneration of catalyst was achieved by activating the catalyst at $523 \mathrm{~K}$ for $4 \mathrm{~h}$.

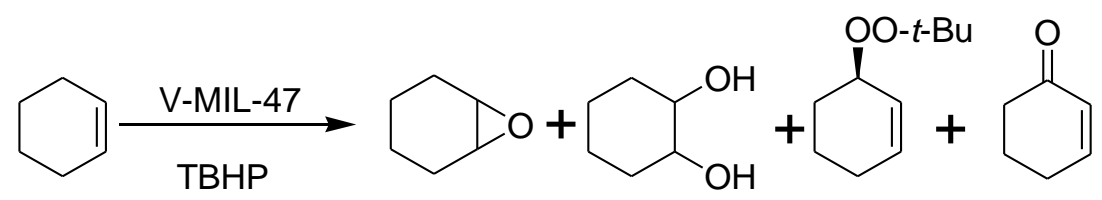

Scheme 6. Oxidation of cyclohexene catalyzed by V-MIL-47 using TBHP as oxidant.

Recently, MIL-101 has been reported as heterogeneous catalyst for the allylic oxidation of alkenes using TBHP as oxidant. ${ }^{64}$ Although epoxidation of alkenes is a process of large industrial importance, allylic oxidation leading to the formation of $\alpha, \beta$ enones or allylic alcohols is a very interesting reaction from the synthetic point of view. $\alpha, \beta$-Enones can undergo Michael additions with a large variety of nucleophiles and are versatile synthetic intermediates. Under the optimized reaction conditions, cyclohexene showed $81 \%$ conversion with more than $93 \%$ selectivity towards 2-cyclohexenone. 
The temperature of the catalyst activation strongly influenced the yield of ketone. MIL101 showed good stability as evidenced by the detection of less than 1 ppm of chromium by hot filtration test. MIL-101 was reused for five times without much loss in the catalytic activity.

Although organic hydroperoxides are generally more suitable for the oxidation of organic compounds and cycloalkenes due to their solubility in organic solvents, hydrogen peroxide is a less costly oxidizing reagent. Hydrogen peroxide has as a general limitation the lack of solubility in common organic solvents and thus generally the reactions employing hydrogen peroxide are conducted in two phases a fact that complicates considerably diffusion and mass transfer between phases.

However, there are examples of the use of hydrogen peroxide in combination with MOFs. Thus, a porous coordination solid of nickel(II) dihydroxyterephthalate has been synthesized by the microwave-assisted method resulting in the formation of $\left[\mathrm{Ni}_{2}\right.$ (dhtp) $\left.\left(\mathrm{H}_{2} \mathrm{O}\right)_{2}\right] \cdot 8 \mathrm{H}_{2} \mathrm{O}$ (where, dhtp $=$ 2,5-dihydroxyterephthalate). [Ni $\mathrm{Ni}_{2}$ (dhtp) $\left.\left(\mathrm{H}_{2} \mathrm{O}\right)_{2}\right] \cdot 8 \mathrm{H}_{2} \mathrm{O}$ has been tested as catalyst for oxidation of cyclohexene using hydrogen peroxide as oxidant. ${ }^{65}$ At equal molar ratio of cyclohexene vs hydrogen peroxide, $7.8 \%$ conversion was observed with $34.7 \%$ cyclohexene oxide, $21.7 \%$ cyclohexenone and $43.6 \%$ of 2-cyclohexenol. Increasing the molar ratio of cyclohexene vs hydrogen peroxide to $3,75.4 \%$ conversion was achieved, decreasing the sectivity of 2cyclohexenol and the formation of $14.4 \%$ of 1,2-cyclohexanediol. ${ }^{65}$ The efficiency of utilization of hydrogen peroxide in the oxidation reaction has also been measured and determined that about $100 \%$ of the consumed hydrogen peroxide participates in cyclohexene oxidation. This fact is remarkable considering the tendency of hydrogen peroxide to undergo disproportionation to water and oxygen. The leaching experiment was performed with 3 eq of hydrogen peroxide and the catalyst showed leaching (the 
percentage not mentioned) at $65{ }^{\circ} \mathrm{C}$ while at room temperature no leaching of nickel was observed.

Besides exploiting the catalytic activity of constitutional metals present in MOFs, these porous materials can also be used to include in the intracrystalline space some guests that can be the catalytic sites for the oxidation. Following a similar work as the one reported previously for ethylbenzene oxidation, polyoxotungstates $\left[\mathrm{PW}_{4} \mathrm{O}_{24}\right]^{3-}$ $\left(\mathrm{PW}_{4}\right)$ and $\left[\mathrm{PW}_{12} \mathrm{O}_{40}\right]^{3-}\left(\mathrm{PW}_{12}\right)$ have been inserted electrostatically into the nanocages of the metal organic framework MIL-101 at a loading between 5 and $14 \mathrm{wt} \%$ and used as catalyst for cyclohexene oxidation. ${ }^{66}$ With 2 equiv of $\mathrm{H}_{2} \mathrm{O}_{2}, 5 \% \mathrm{PW} / \mathrm{MIL}-101$ showed $76 \%$ substrate conversion after $3 \mathrm{~h}$ with $74 \%$ selectivity of cyclohexene oxide. Very similar results in terms of conversion and cyclohexene oxide selectivity were achieved for $5 \% \mathrm{PW}_{12} / \mathrm{MIL}-101$. The allylic oxidation products, 2-cyclohexene-1-ol and 2cyclohexene-1-one (totally ca. 10\%) were identified as the main byproducts, while the product of epoxide ring-opening, 1,2-trans-cyclohexane diol, was not found in the reaction mixture. This contrasts with the homogeneously catalyzed oxidation in the presence of $\mathrm{PW}_{4}$ or $\mathrm{PW}_{12}$ where the diol was the main byproduct. Elemental analysis and hot filtration test showed the absence of any tungsten (from the polyoxotungstates) or chromium (from MIL-101) traces in the liquid phase and that the reaction stops when the solid is removed. $\mathrm{PW}_{4} / \mathrm{MIL}-101$ showed a slight decrease in the epoxide yield after three runs and this could be attributed to the decrease in the specific surface area and pore volume. Powder XRD shows amorphization of the MIL-101 catalyst due to partial decomposition during the cyclohexene oxidation with hydrogen peroxide. ${ }^{66}$

In continuous effort in synthesizing new porous MOFs, Hupp and coworkers reported the synthesis of a porous organic polymer ${ }^{67}$ (POP) containing free-base porphyrin subunits by the condensation of a bis(phthalic acid)porphyrin with tetra(4- 
aminophenyl)methane. Metallation by post-synthesis modification affords microporous materials incorporating either Fe or Mn(porphyrins) that have been shown to be active catalysts for styrene epoxidation using iodosylbenzene as oxidant. ${ }^{67}$ Metallated mesotetrapentafluorophenylporphyrin (TPFPP) as homogenous complexes with $\mathrm{Mn}$ and Fe rapidly produce styrene epoxides, but with facile deactivation and degradation of the catalyst reaching maximum TON of 780 in the case of (TPFPP)Mn, and 170 in the case of (TPFPP)Fe. In contrast, both the Fe- and Mn-PPOP show greater stability than the analogous homogenous catalysts and Mn-PPOP is active for more than 600 turnovers and Fe-PPOP with 300 without displaying any sign of decomposition. Although both catalysts were reused for three cycles, the activity was decreased in the second and third cycle. The loss of catalytic activity can be attributed to the oxidation of individual pyrrolic rings and not to the destruction of the polymer network as evidenced by SEM analysis of the spent catalyst. Demetallation of the material was also ruled out as ICP analysis showed the same metal content before and after catalytic oxidation.

In the previous examples, the oxidzing reagents have been organic hydroperoxides and occassionally hydrogen peroxide and iodoso compounds. Hydroperoxides are environmentally benign but it would be more desirable to use directly molecular oxygen as oxidant. The control of aerobic oxidations harrnessing the reactivity to the desired products is a challenge in catalysis. In this context, $\left[\mathrm{Cu}(\right.$ bpy $\left.)\left(\mathrm{H}_{2} \mathrm{O}\right)_{2}\left(\mathrm{BF}_{4}\right)_{2}(\mathrm{bpy})\right]$ (bpy: 4,4'-bipyridine) has been reported as heterogeneous catalyst for allylic oxidation of cyclohexene in the presence of oxygen as oxidant. ${ }^{68}$ In all the cases, the selectivity of 2-cyclohexenylhydroperoxide was more than $85 \%$ with only detectable amount of cyclohexene epoxide, 2-cyclohexenone and 2-cyclohexenol.

As it is commented earlier, the use of molecular oxygen as terminal oxidant is always desirable and in this connection, a manganese(II) acetylacetonate complex has 
been immobilized to the amino functionalized isoreticular metal-organic framework IRMOF-3 through a one-step post-synthetic route providing heterogeneous catalyst IRMOF-3(Mn). ${ }^{69}$ This catalyst has been tested for the epoxidation of cyclohexene, cyclooctene and styrene using pivalaldehyde and oxygen as oxidant in toluene medium (Scheme 7). Cyclohexene showed $68 \%$ of conversion towards $92 \%$ of cyclohexene oxide accompained with $8 \%$ of allylic products. Cyclooctene reached $60 \%$ conversion exhibiting $96 \%$ epoxide selectivity. Analogously, styrene showed $52 \%$ conversion with $80 \%$ selectivity towards styrene oxide. IRMOF-3(Mn) was reused for three cycles with a similar reactivity and selectivity in all the three substrates. Powder XRD showed no sign of decomposition of Mn complex in the spent catalyst after the catalytic oxidation reaction and no leaching of Mn observed. The major concern is the need of 2 equivalent of pivalaldehyde and no mechanism for the high selectivity observed was proposed.

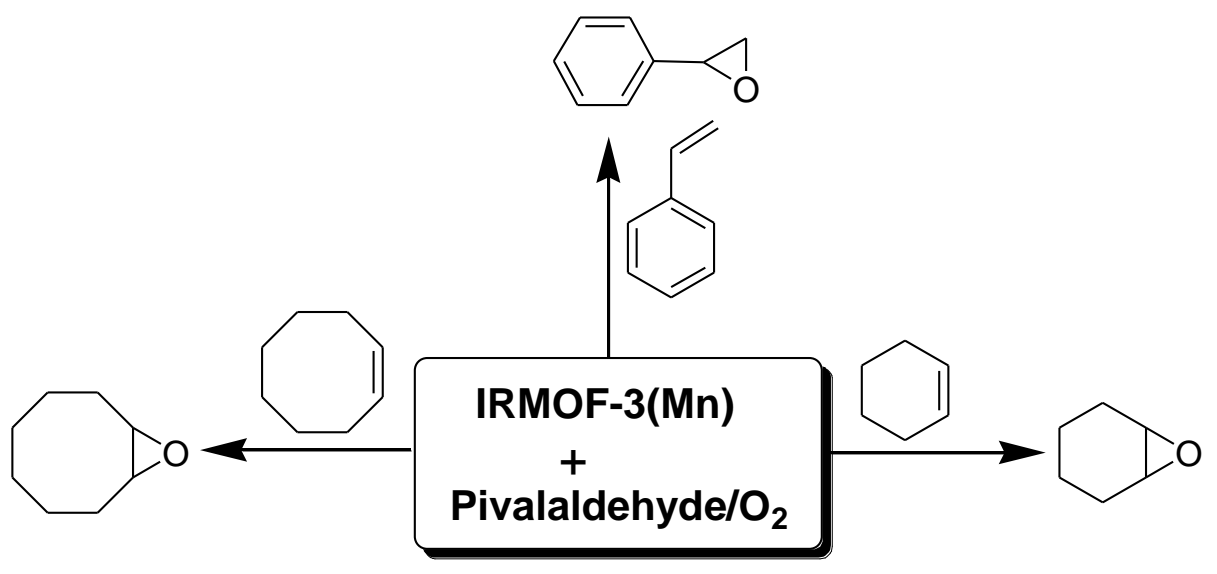

Scheme 7. Epoxidation of olefins catalyzed by IRMOF-3(Mn) with pivalaldehyde/ $\mathrm{O}_{2}$ as oxidant.

After having observed a high catalytic activity for cyclooctane and benzyl amine oxidation, we were interested to make use of $\mathrm{NHPI} / \mathrm{Fe}(\mathrm{BTC})$ as catalyst for cycloalkene oxidation. In this context, epoxidation of cyclooctene was performed using NHPI/Fe(BTC) as catalyst under neat condition at $100{ }^{\circ} \mathrm{C} .{ }^{70}$ This catalyst showed $9 \%$ 
conversion with $95 \%$ selectivity towards epoxide. An effort to increase the conversion to $48 \%$ reduced the selectivity of epoxide to $85 \%$. Even though, this selectivity is still remarkable at such high conversion in the absence of solvent. Also, it has been demonstrated that the ring size plays an important role in determining the conversion and selectivity of the products. Thus, cyclopentene and cyclohexene undergo preferentially allylic oxidation under the same conditions in which epoxidation occurs for cyclooctene. The catalyst could be reused two times and its structural integrity was determined from powder XRD by comparing fresh and spent catalyst.

In contrast to the current state of the use of MOFs for oxidation of alkenes using oxygen, there is a lack in the literature reporting the use of zeolites for aerobic oxidation of alkenes. This is due to the fact that aluminosilicates are inert as oxidation catalyst and the presence of suitable transition metals is needed. However, as we have commented, transition metals in zeolites tend to evolve from framework to extra framework positions, rendering unstable materials whose catalytic activity vary depending on the state of the catalytic sites.

\section{Oxidation of alcohols}

Selective oxidation of primary alcohols to aldehydes is a highly relevant transformation in organic chemistry due to the properties and chemical reactivity of these carbonylic compounds that make aldehydes as starting materials in many synthetic methods. In this connection, developing a suitable catalyst for this oxidation is a long standing reasearch area. $\left[\operatorname{Pd}(2-\text { pymo })_{2}\right]_{n}(2 \text {-pymo }=2 \text {-hydroxypyrimidinolate })^{71}$ has been synthesised with $32 \%$ wt of Pd and used for the selective aerobic oxidation of cinnamyl alcohol reaching $74 \%$ of selectivity towards the desired cinnamaldehyde in $20 \mathrm{~h}$. Besides the applications and uses of cinnamaldehydes, oxidation of cinnamyl alcohol is considered a model reaction since there is an array of competitive pathways 
that can take place simaltaneously to the alcohol oxidation including epoxidations, $\mathrm{C}=\mathrm{C}$ isomerization and even polymerization and hydrogenation. Hot filtration test did not show the presence of $\mathrm{Pd}$ and hence stability of the catalyst was assumed. However, since palladium in homogeneous phase can be highly active even in very low concentrations and trace amounts, ${ }^{72,73}$ the possibility that $\operatorname{Pd}(2 \text {-pymo })_{2}$ is acting as solid reservoir providing some palladium to the solution was not conclusively ruled out. It has also been shown that the $\operatorname{Pd}(2 \text {-pymo })_{2}$ catalyst is active for Suzuki cross coupling and for the shape selective hydrogenation of olefins. ${ }^{71}$

$\left[\mathrm{Zn}_{4} \mathrm{O}(\mathrm{btb})_{2}\right]_{8}\left(\mathrm{H}_{3}\right.$ btb $=$ 1,3,5-benzenetribenzoic acid $)$, MOF-177 has been used as support for loading of $\mathrm{Pt}$ nanoparticles in the range of $2-5 \mathrm{~nm}$ and used them as catalyst for hydrogen storage and aerobic oxidation of benzylic and allylic alcohols at room temperature under base and solvent free conditions. ${ }^{74}$ Benzylic susbstrates showed very good reactivity with TON > 500 (Scheme 8 ) and cinnamyl alcohol with 385 . In contrast, aerobic oxidation of iodo and bromo substituted benzyl alcohols failed due to their steric hindrance that impedes these substrates to reach the active sites. Aliphatic alcohols were found to be unreactive under identical conditions. After using MOF-177 as catalyst for the aerobic oxidation of benzyl alcohol, powder XRD of the used material revealed that breakdown of MOF host lattice occurred leading to a negligible conversion of benzyl alcohol in an attempted reuse. This lack of MOF-177 stability could be anticipated from the fact that $\mathrm{Zn}_{4} \mathrm{O}$ cluster is highly water sensitive and water is produced as byproduct during the oxidation.

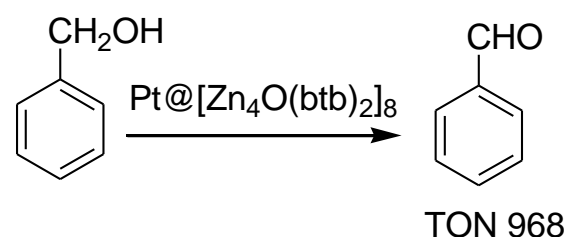


Scheme 8. Aerobic oxidation of benzyl alcohol to benzaldehyde using Pt nanoparticles supported on $\left[\mathrm{Zn}_{4} \mathrm{O}(\mathrm{btb})_{2}\right]_{8}$.

Recently, Haruta and co-workers deposited Au clusters on several MOFs such as MOF-5, $\left[\mathrm{Zn}_{4} \mathrm{O}(\mathrm{bdc})_{3}\right]_{\mathrm{n}}$ (bdc=benzene-1,4-dicarboxylate), Al-MIL-53, $[\mathrm{Al}(\mathrm{OH})(\mathrm{bdc})]_{\mathrm{n}}$ and $\mathrm{Cu}_{3}(\mathrm{BTC})_{2}$. Volatile organogold complex, $\mathrm{Me}_{2} \mathrm{Au}(\mathrm{acac})$ (acac=acetylacetonate) and MOFs were ground in an agate mortar in air for $20 \mathrm{~min}$ at room temperature. Then, the mixture was treated in a $\mathrm{N}_{2}$ stream having $10 \mathrm{vol} \% \mathrm{H}_{2}$ at $120{ }^{\circ} \mathrm{C}$ for $2 \mathrm{~h}$ to obtain $\mathrm{Au}$ supported on different MOFs. The very simple grinding procedure for gold incorporation is remarkable. These gold containing catalysts were tested for the liquid phase aerobic oxidation of benzyl alcohol and 1-phenylethanol in methanol. ${ }^{75}$ Au/MOF5 exhibited $82 \%$ conversion of benzyl alcohol in the presence of base resulting in the formation of $66 \%$ of methyl benzoate and $3 \%$ benzaldehyde while in the absence of base, $69 \%$ conversion with $23 \%$ methyl benzoate and $31 \%$ benzaldehyde. Au/AlMIL-53 showed $98 \%$ conversion of benzyl alcohol with the selectivity of $2 \%$ of benzaldehyde and $77 \%$ of methyl benzoate. ${ }^{75}$ Gold nanoparticles supported on MOF-5 $(0.5$ wt $\%$ Au with a mean diameter of $4.8 \pm 2.2 \mathrm{~nm})$ exhibited the highest catalytic activity among all the $\mathrm{Au}$ supported on different MOFs by solid grinding for 1phenylethanol oxidation in the presence of base affording $79 \%$ yield of acetophenone. $^{75}$

Recently, nanoparticles of gold has been supported also on MIL-101 using different methods and Au deposited by colloidal deposition with PVP as protecting agent was the sample exhibiting the highest catalytic activity for aerobic oxidation of alcohols to aldehydes under base free conditions. ${ }^{76}$ This may be due to lower mean diameter of gold nanoparticles $(2.3 \mathrm{~nm})$ compared to other methods of loading on MIL101. However, how diffusion of gold nanoparticles inside MOF occurs is a matter that 
deserves further study. Benzylic alcohols were oxidized to their corresponding aldehydes in high conversion $(>95 \%)$ and selectivity $(>99 \%) .{ }^{76}$ Secondary aliphatic alcohols showed higher activity than primary counterparts (Scheme 9). Alcohols with heteroatom were also smoothly oxidized with high conversion and selectivity. The catalyst showed no leaching of gold and TEM analysis confirmed that particle size of gold before and after oxidation reaction remains without aggregation. The catalyst showed consistent yield (> $99 \%$ ) for the oxidation of 4-methoxybenzyl alcohol until sixth cycle. This catalytic system showed very high TOF for the aerobic oxidation of 1phenylethanol with $29300 \mathrm{~h}^{-1} .^{76}$ This high catalytic activity is probably due to the high dispersion of Au nanoparticles in combination with a beneficial synergetic effect of the MIL-101 support. As is well-known, the support may play an important role, either direct or indirect, in determining the activity and selectivity of gold. ${ }^{77}$ In addition, the higher catalytic activity of this gold-containing MOF could be explained from the fact of good chemical resistance to water and organic solvents by MIL-101 and the larger pore size of MIL-101 (ca. $30 \AA$ ) facilitating mass transfer of reactant/product molecules inside the pores.

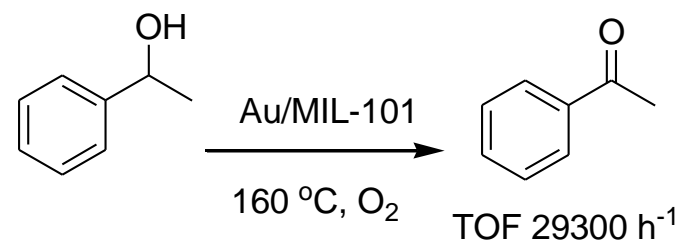

Scheme 9. Aerobic oxidation of 1-phenylethanol catalyzed by Au/MIL-101 .

Aerobic oxidation of benzyl alcohols has been reported using $\mathrm{Cu}_{3}(\mathrm{BTC})_{2}$ as catalyst in the presence of base and catalytic amount of TEMPO in acetonitrile medium (Scheme 10). ${ }^{78}$ Although this catalyst showed moderate catalytic activity for benzyl alcohols, they exhibited low activity towards the oxidation of aliphatic, secondary and cyclic alcohols. In addition to the lack of general scope of $\mathrm{Cu}_{3}(\mathrm{BTC})_{2}$ in combination 
with TEMPO, leaching test showed $1 \mathrm{ppm}$ of copper and the catalyst undergoes some changes in its crystal structure as evidenced by XRD. It has been demonstrated that benzoic acid formed during the reaction could lead to deactivation of catalyst by coordination with the free metal centres. Reactivation is certainly an interesting issue. Considering that the structure of common MOFs are based on metal-carboxylate interactions it may happen that carboxylic acids are general poisons for any reaction requiring free coordination sites of framework nodal metals by strong interaction with the metal sites. Since carboxylic acids can generally be formed at least in minor amounts in the aerobic oxidation of primary alcohols, it is important to show if the poisoning by the presence of carboxylic acids is a general deactivation mechanism.

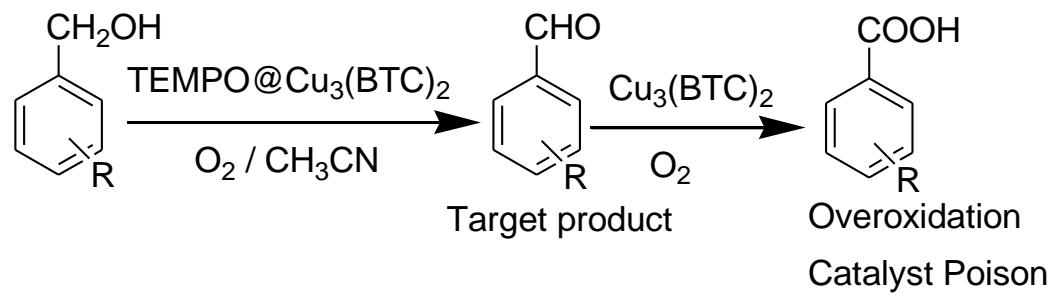

Scheme 10. Deactivation of $\mathrm{Cu}_{3}(\mathrm{BTC})_{2}$ by over oxidation of benzaldehyde.

\section{Enantioselective oxidation by chiral MOFs}

MOFs are constituted by nodal metallic ions or clusters connected by rigid linkers. As we have commented in comparison with zeolites, the organic components allow large flexibility in design and modification of the linker. One special case is when the linker exhibits chirality and only one of the enantiomers is used in the preparation of MOFs. Depending on the synthesis conditions homochiral MOFs are obtained that can be suitable as solid catalysts to transmit chirality starting from a chiral substrates (asymmetric induction by catalysis). ${ }^{33}$ The field of chiral MOFs has recently reviewed and among the chiral linkers that has been reported so far the most widely used are those based on 1,1'-binaphthyls having substituents in the periphery to coordinate with 
metallic nodes. Also, one remarkable example in which a chiral metal salen complex has been used as linker has been reported by the group of Hupp. ${ }^{79}$ Scheme 11 shows some of the chiral linkers that have been employed for the synthesis of chiral MOFs.
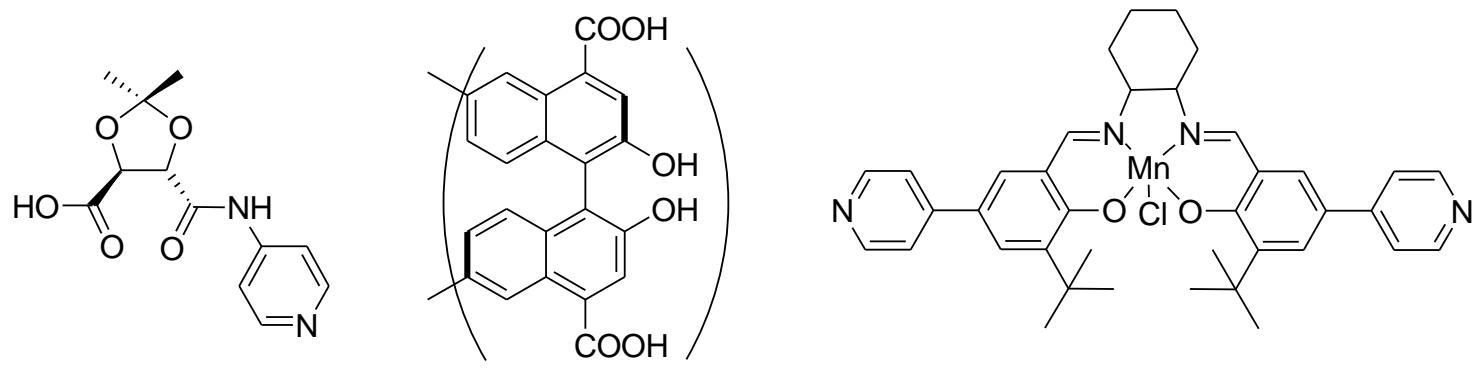

Scheme 11. Chiral linkers used for the synthesis of chiral MOFs

Besides the use of chiral linkers, another possible strategy to develop asymmetric MOF catalysts could be to start with a chiral MOF and subsequently modify it with chiral spectators accommodated inside the MOF cavities.

In is interesting to compare this situation with zeolites and mesoporous silicas. The synthesis of homochiral zeolites has been a target in the 1990s and even now and not much success has been achieved. ${ }^{80}$ Zeolites require the use of an organic template and by using chiral organic templates, it was expected to obtain homochiral zeolites. However, the harsh conditions (high temperature, strongly basic media, long reaction times etc) required for the synthesis of zeolites has resulted in poor results. Since chiral zeolites are difficult to obtain, the possibilities to include chiral spectators inside zeolite cavities has been more frequently studied but also the enantiomeric excesses obtained have been very low. ${ }^{81}$

Much better results have been obtained starting with mesoporous silica or organosilicas and introducing in the inorganic framework chiral organic components or 
metallic complexes to produce chiral solids. ${ }^{82,83}$ Particular successful has been the development of chiral periodic mesoporous organosilicas (ChiMO). ${ }^{84}$ This strategy however has the disadvantage of dedicated organic synthesis to obtain chiral silanes needed for the hybrid organosilica and subsequent use of organic surfactant (that later has to be removed) in the synthesis of the mesoporous solids. In recent times, chiral MOFs have been successfully reported as catalysts for many enantioselective reactions including epoxidation of alkenes. ${ }^{85-87}$

Asymmetric induction typically are carried out at low temperatures and under mild conditions. ${ }^{88}$ In fact, it is very frequently observed that ambient temperature or the presence of acids and bases spoils enantiomeric excess. These mild conditions are in fact very well suited to preserve the crystal structure of MOFs and in this regard reaction conditions are compatible with MOF stability. The key point is being again diffusion of reagents and products through the pores in liquid phase.

Concerning enantioselective oxidations, one key point is the reagents that are most commonly employed. Typically the reagent should transfer oxygen to the active catalytic site in such a way that only one enantiomer of the active site is formed. This generally requires the use of bulky reagents. Thus, although hypochlorite and hydroperoxides have sometimes being used for oxidations other reagents such as $\mathrm{N}$ oxides and iodoso compounds are more often used.

The selection of this type of bulky oxidant such as iodosylbenzene for enantioselective oxidation is not very fortunate from the "green point of view" since this is a stoichiometric reagent that would generate a large amount of waste. However, due to the influence of steric hindrance in the asymmetric induction, it is almost impossible to induce chirality using molecular oxygen as the direct oxidant. Nevertheless, a 
catalytic cycle in which under stoichiometric amounts of iodosylbenzene are used and its reoxidation is performed by oxygen could be feasible.

Besides reagents another point of interest is the type of products that would be of industrial interest. In this sense, chiral epoxides are one of the preferred targets since the reactivity of these compounds allows later to transmit chirality to a large variety of mono and difunctional compounds. ${ }^{89}$ It is worth commenting that chiral epoxidations of allylic alcohols reported by Sharpless using homogeneous chiral titanium complexes as catalysts constituted a milestone in this area ${ }^{90}$ As an example of how chiral MOFs can be used as catalysts for alkene epoxidation, Nguyen and coworkers studied the enantioselective epoxidation of chromenes. In this example, Mn(III)-Schiff base complex forming part of the MOF linker in $\mathrm{Zn}_{2}(\mathrm{bpdc})_{2} \mathrm{~L} .10 \mathrm{DMF} .8 \mathrm{H}_{2} \mathrm{O}^{91}$ ( $\mathrm{L}=(\mathrm{R}, \mathrm{R})-(-)-$ 1,2-cyclohexanediamino-N,N'-bis(3-tert-butyl-5-(4-pyridyl)salicylidene) $\mathrm{Mn}^{\mathrm{III}} \mathrm{Cl}$ and bpdc $=$ biphenyldicarboxylate) was used as solid catalyst (Scheme 12). ${ }^{91}$ Although, L as homogeneous catalyst showed higher initial activity than $\mathrm{Zn}_{2}(\text { bpdc })_{2} \mathrm{~L} .10 \mathrm{DMF} \cdot 8 \mathrm{H}_{2} \mathrm{O}$, at long reaction times the latter solid showed better activity by exhibiting higher turnover number (TON) of 1430 with $82 \%$ ee while L showed $88 \%$ ee with TON of around 600. Under these experimental conditions, leaching of Mn was determined to occur between 4 and $7 \%$ in each cycle. This loss of Mn has been proposed to be responsible for the decrease of yield and TON observed for $\mathrm{Zn}_{2}(\text { bpdc })_{2} \mathrm{~L} .10 \mathrm{DMF} .8 \mathrm{H}_{2} \mathrm{O}$ upon reuse. The gradual decrease in catalytic activity is not accompanied by a decrease in $e e .^{91}$

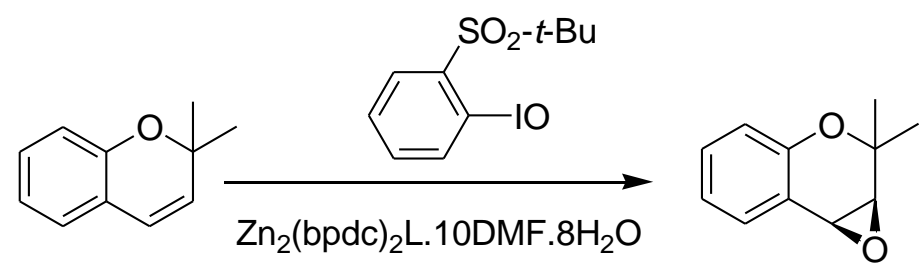


Scheme 12. Enantioselective epoxidation of 2,2-dimethyl-2H-chromene by 2-tbutylsulfonyliodobenzene as oxidant using $\mathrm{Zn}_{2}(\text { bpdc) })_{2} \mathrm{~L} .10 \mathrm{DMF} .8 \mathrm{H}_{2} \mathrm{O}$ as heterogeneous catalyst.

The reader is referred to recent reviews ${ }^{33}$ on chiral MOFs for a more complete coverage on this topic but for the present purpose, we should stress that a methodology in which molecular oxygen or organic hydroperoxides are the final oxidants and some chiral cocatalysts is used in small percentages is still to be developed and will be significant development in the field of catalytic enantioselective oxidations.

\section{Conclusions and future perspectives}

From the above examples on the use of MOFs as heterogeneous catalysts for oxidation reactions it can be concluded that these materials are going to be widely used for liquid phase reactions expanding considerably the range of organic compounds that will be selectively oxidized. All these works should demonstrate conclusively the stability of the framework and if possible they should address the deactivation mechanism. General statements like observing the same catalytic behaviour for a few additional cycles is not sufficient. Temporal profiles after several uses should be provided showing not only conversion data at a given time but also initial reaction rates. In addition long term stability would be better checked by performing continuous flow experiments rather than batch reactions. One figure of merit that would be interesting to know is maximum productivity before the MOFs becomes deactivated and the reactivation procedures. Also, it would be of large interest, for those cases in which real industrial applications could be envisioned, to determine which are the products derived from the organic linker under the reaction conditions even if this linker oxidation requires very long time. 
Concerning the oxidizing reagents and even though organic hydroperoxides are simpler to be used due to solubility, more efforts should be devoted to carry out oxidations with hydrogen peroxide. This certainly requires the use of MOFs that are stable in the presence of water, a fact that is still not general in many cases. Also, it is easily anticipated that oxidation using molecular oxygen in combination of MOFs as catalysts is a field that will develop intensively in the near future. In this area besides the use of nodal metals a strategy in which micropores contain metal nanoparticles such as gold or palladium seems very promising. Also, the combination of MOFs with organic promoters of radical chain mechanisms have large potential in aerobic oxidations.

Concerning the substrates, it would be highly relevant to apply MOFs for oxidation of cyclohexane, epoxidation of propene and oxidation of linear alcohols in non aqueous medium. In these three targets, there is no doubt that MOFs, sometimes following the lead of previous work with zeolites are very promising as solid catalysts.

Finally considering the easy design and simplicity of the synthesis of chiral MOFs there is no doubt that in the near future, this field will grow enormously, also for oxidation reactions and some excellent chiral solid catalysts will be developed in the near future.

\section{Acknowledgements}

Financial support by the Spanish DGI (CTQ2009-11587 and CTQ2010-18671) is gratefully acknowledged. Funding of European Commission through an integrated project (MACADEMIA) is also acknowledged.

\section{References}

1. Z. Wang, G. Chen and K. Ding, Chem. Rev, 2009, 109, 322. 
2. J. Y. Lee, O. K. Farha, J. Roberts, K. A. Scheidt, S. T. Nguyen and J. T. Hupp, Chem. Soc. Rev, 2009, 38, 1450.

3. A. Corma, H. Garcia and F. X. Llabres i Xamena, Chem. Rev, 2010, 110, 4606.

4. O. M. Yaghi, M. O'Keeffe, N. W. Ockwig, H. K. Chae, M. Eddaoudi and J. Kim, Nature, 2003, 423, 705-714.

5. H. Furukawa, N. Ko, Y. B. Go, N. Aratani, S. B. Choi, E. Choi, A. O. Yazaydin, R. Q. Snurr, M. O'Keeffe, J. Kim and O. M. Yaghi, Science, 2010, 239, 424 428

6. S. Kitagawa, R. Kitaura and S. Noro, Angew. Chem. Int. Ed., 2004, 43, 23342375.

7. G. Ferey, Chem. Soc. Rev, 2008, 37, 191-214.

8. M. Kondo, T. Okubo, A. Asami, S. Noro, T. Yoshitomi, S. Kitagawa, T. Ishii, H. Matsuzaka and K. Seki, Angew. Chem., Int. Ed., 1999, 38, 140-143.

9. H. Furukawa and O. M. Yaghi, J. Am. Chem. Soc, 2009, 25, 8876-8883.

10. J. L. C. Rowsell, A. R. Millward, K. S. Park and O. M. Yaghi, J. Am. Chem. Soc, 2004, 126, 5666-5667.

11. M. Eddaoudi, H. Li and O. M. Yaghi, J. Am. Chem. Soc., 2000, 122, 1391-1397.

12. R. Kitaura, K. Seki, G. Akiyama and S. Kitagawa, Angew. Chem., Int. Ed., 2003, 42, 428-431.

13. N. L. Rosi, J. Eckert, M. Eddaoudi, D. T. Vodak, J. Kim, M. O'Keeffe and O. M. Yaghi, Science, 2003, 300, 1127-1129.

14. L. J. Murray, M. Dincă and J. R. Long, Chem. Soc. Rev, 2009, 38 1294-1314

15. G. Ferey, Nature, 2005, 436, 187-188.

16. D. Farrusseng, S. Aguado and C. Pinel, Angew. Chem. Int. Ed., 2009, 48, 7502.

17. A. Dhakshinamoorthy, M. Alvaro and H. Garcia, Chem. Eur. J., 2010, 16, 8530.

18. A. Dhakshinamoorthy, M. Alvaro and H. Garcia, Adv. Synth. Catal., 2010, 352, 711.

19. A. Dhakshinamoorthy, M. Alvaro and H. Garcia, Adv. Synth. Catal., 2009, 351, 2271.

20. A. Dhakshinamoorthy, M. Alvaro and H. Garcia, Chem. Commun., 2010, 46, 6476-6478.

21. F. Gándara, B. Gomez-Lor, E. Gutiérrez-Puebla, M. Iglesias, M. A. Monge, D. M. Proserpio and N. Snejko, 2008, 20, 72-76.

22. K. Schlichte, T. Kratzke and S. Kaskel, Microporous Mesoporous

Mater. , 2004, 73, 81.

23. L. Alaerts, E. Seguin, H. Poelman, F. Thibault-Starzyk, P. A. Jacobs and D. E. De Vos, Chem.sEur. J, 2006, 12, 7353.

24. B. Gomez-Lor, E. Gutierrez-Puebla, M. Iglesias, M. A. Monge, C. Ruiz-Valero and N. Snejko, Inorg. Chem., 2002, 41, 2429.

25. K. S. Suslick, P. Bhyrappa, J. H. Chou, M. E. Kosal, S. Nakagaki, D. W. Smithenry and S. R. Wilson, Acc. Chem. Res, 2005, 38, 283.

26. C. N. Kato, M. Hasegawa, T. Sato, A. Yoshizawa, T. Inoue and W. Mori, J. Catal., 2005, 230, 226.

27. M. Ranocchiari and J. A. van Bokhoven, Phys. Chem. Chem. Phys, 2011, 2394a.

28. F. Vermoortele, R. Ameloot, A. Vimont, C. Serre and D. De Vos, 2011, 47, 1521-1523.

29. A. Corma, in Recent Advances in the Science and Technology of Zeolites and Related Materials, Pts $a$ - C, eds. E. VanSteen, M. Claeys and L. H. Callanan, 2004, vol. 154, pp. 25-40. 
30. J. L. Sun, C. Bonneau, A. Cantin, A. Corma, M. J. Diaz-Cabanas, M. Moliner, D. L. Zhang, M. R. Li and X. D. Zou, Nature, 2009, 458, 1154-U1190.

31. A. Philippaerts, S. Paulussen, A. Breesch, S. Turner, O. I. Lebedev, G. V. Tendeloo, B. Sels and P. A. Jacobs, Angew. Chem. Int. Ed., 2011, 50, 10.1002/anie.201007513.

32. T. Tatsumi, H. Sakashita and K. Asano, J . Chem. Soc., Chem. Commun, 1993, 1264-1265.

33. W. Lin, Top. Catal, 2010, 53, 869-875.

34. Y. Gao and K. B. Sharpless, J. Org. Chem, 1988, 53, 4081-4084.

35. C. W. Jones, Applications of Hydrogen Peroxide and Derivatives (Royal Society of Chemistry, Thomas Graham House, London)

1999.

36. A. Corma and H. Garcia, Chem. Rev, 2002, 102, 3837-3892.

37. G. Bellussi, A. Carati, M. G. Clerici, A. Esposito, R. Millini and F. Buonomo, 1989, Belg. Pat. 1001038

38. M. Taramasso, G. Perego and B. Notari, 1983, US 4410501.

39. P. T. Anastas and J. C. Warner, Green Chemistry: Theory and Practice, Oxford University Press: New York, 1998, , p.30.

40. A. Fatiadi, Synthesis, 1987, 85-127.

41. E. N. Jacobsen, In Catalytic Asymmetric Synthesis, Ojima, I., Ed.; VCH: New York,

, 1993, 159-202.

42. C. Di Nicola, Y. Yu. Karabach, A. M. Kirillov, M. Monari, L. Pandolfo, C. Pettinari and A. J. L. Pombeiro, Inorg. Chem., 2007, 46, 221-230.

43. P. Horcajada, S. Surble, C. Serre, D. Y. Hong, Y. K. Seo, J. S. Chang, J. M. Greneche, I. Margiolaki and G. Ferey, Chem. Commun., 2007, 2820-2822.

44. A. Dhakshinamoorthy, M. Alvaro and H. Garcia, Chem. Eur. J., 2011.

45. I. Hermans, J. Van Deun, K. Houthoofd, J. Peeters and P. A. Jacobs, J. Catal. , 2007, 251, 204-212.

46. K. Weissermel and H.-J. Harpe, Industrial Organic Chemistry, 4th edn., WileyVCH, Weinheim, 2003.

47. J. Kim, S. Bhattacharjee, K.-E. Jeong, S.-Y. Jeong and W.-S. Ahn, Chem. Commun., 2009, 3904-3906.

48. A. Dhakshinamoorthy, M. Alvaro and H. Garcia, J. Catal., 2009, 267, 1-4.

49. F. X. Llabrés i Xamena, O. Casanova, R. Galiasso Tailleur, H. Garcia and A. Corma, J. Catal., 2008, 255, 220-227.

50. F. Yu, P.-Q. Zheng, Y.-X. Long, Y.-P. Ren, X.-J. Kong, L.-S. Long, Y.-Z. Yuan, R.-B. Huang and L.-S. Zheng, Eur. J. Inorg. Chem., 2010, 4526-4531.

51. A. B. Sorokin, J.-L. Seris and B. Meunier, Science, 1995, 268, 1163.

52. O. V. Zalomaeva, A. B. Sorokin and O. A. Kholdeeva, Green Chem, 2010, 12, 1076-1082.

53. O. V. Zalomaeva, K. A. Kovalenko, Y. A. Chesalov, M. S. Mel'gunov, V. I. Zaikovskii, V. V. Kaichev, A. B. Sorokin, O. A. Kholdeeva and V. P. Fedin, Dalton Trans., 2011, c0dt01474e.

54. E. Kockrick, T. Lescouet, E. V. Kudrik, A. B. Sorokin and D. Farrusseng, Chem. Commun., 2011, 47, 1562-1564.

55. K. Brown, S. Zolezzi, P. Aguirre, D. Venegas-Yazigi, V. Paredes-Garcia, R. Baggio, N. M. A.; and E. Spodine, Dalton Trans., 2009, 1422-1427. 
56. A. Corma, M. T. Navarro and J. Perez-Pariente, J. Chem. Soc. Chem. Commun., 1994, 147.

57. A. Corma, U. Diaz, M. E. Domine and V. Fornes, Chem Commun, 2000, 137138.

58. A. Corma and H. García, Chem. Rev., 2002, 102, 3837-3892.

59. A. Corma, P. Esteve, A. Martinez and S. Valencia, J. Catal., 1995, 152, 18-24.

60. A. Corma, M. E. Domine, J. A. Gaona, J. L. Jorda, M. T. Navarro, F. Rey, J. Perez-Pariente, J. Tsuji, B. McCulloch and L. T. Nemeth, Chem. Commun., 1998, 2211.

61. A. Corma, J. L. Jorda, M. T. Navarro and F. Rey, Chem. Commun., 1998, 18991900.

62. M. Tonigold, Y. Lu, B. Bredenkotter, B. Rieger, S. Bahnmuller, J. Hitzbleck, G. Langstein and D. Volkmer, Angew. Chem. Int. Ed., 2009, 48, 7546-7550.

63. K. Leus, I. Muylaert, M. Vandichel, G. B. Marin, M. Waroquier, V. V. Speybroeck and P. V. Der Voort, Chem. Commun., 2010, 46, 5085-5087.

64. N. V. Maksimchuk, K. A. Kovalenko, V. P. Fedin and O. A. Kholdeeva, $A d v$. Synth. Catal., 2010, 352, 2943-2948.

65. J. S. Lee, S. B. Halligudi, N. H. Jang, D. W. Hwang, J.-S. Chang and Y. K. Hwang, Bull. Korean Chem. Soc., 2010, 31, 1489-1495.

66. N. V. Maksimchuk, K. A. Kovalenko, S. S. Arzumanov, Y. A. Chesalov, M. S. Melgunov, A. G. Stepanov, V. P. Fedin and O. A. Kholdeeva, Inorg. Chem., 2010, 49, 2920-2930.

67. A. M. Shultz, O. K. Farha, J. T. Hupp and S. T. Nguyen, Chem. Sci., 2011, 10.1039/c1030sc00339e.

68. D. Jiang, T. Mallat, D. M. Meier, A. Urakawa and A. Baiker, J. Catal., 2010, 270, 26-33.

69. S. Bhattacharjee, D.-A. Yang and W.-S. Ahn, Chem. Commun., 2011, 10.1039/c1031cc00069a.

70. A. Dhakshinamoorthy, M. Alvaro and H. Garcia, Angew. Chem. Int. Ed., 2011, in reviewing process.

71. F. X. Llabrés i Xamena, A. Abad, A. Corma and H. Garcia, J. Catal., 2007, 250, 294-298.

72. A. H. M. de Vries, J. Mulders, J. H. M. Mommers, H. J. W. Henderickx and J. G. de Vries, Organic Letters, 2003, 5, 3285-3288.

73. M. T. Reetz and J. G. de Vries, Chemical Communications, 2004, 1559-1563.

74. S. Proch, J. Herrmannsdorfer, R. Kempe, C. Kern, A. Jess, L. Seyfarth and J. Senker, Chem. Eur. J., 2008, 14, 8204-8212.

75. T. Ishida, M. Nagaoka, T. Akita and M. Haruta, Chem. Eur. J., 2008, 14, 84568460.

76. H. Liu, Y. Liu, Y. Li, Z. Tang and H. Jiang, J. Phys. Chem. C, 2010, 114, 13362-13369.

77. A. Corma and H. Garcia, Chemical Society Reviews, 2008, 37, 2096-2126.

78. A. Dhakshinamoorthy, M. Alvaro and H. Garcia, ACS Catal., 2011, 1, 48-53.

79. S.-H. Cho, T. Gadzikwa, M. Afshari, S. T. Nguyen and J. T. Hupp, Eur. J. Inorg. Chem. , 2007, 4863-4867.

80. J. H. Yu and R. R. Xu, J. Mater. Chem., 2008, 18, 4021-4030.

81. M. Benitez, G. Bringmann, M. Dreyer, H. Garcia, H. Ihmels, M. Waidelich and K. Wissel, Journal of Organic Chemistry, 2005, 70, 2315-2321.

82. C. Baleizão and H. Garcia, Chem. Rev., 2006, 106, 3987-4043. 
83. M. Alvaro, M. Benitez, D. Das, B. Ferrer and H. Garcia, Chem. Mater., 2004, 16, 2222-2228.

84. C. Baleizão, B. Gigante, D. Das, M. Alvaro, H. Garcia and A. Corma, Chem. Commun., 2003, 1860-1861.

85. M. Banerjee, S. Das, M. Yoon, H. J. Choi, M. H. Hyun, S. M. Park, G. Seo and K. Kim, J. Am. Chem. Soc, 2009, 131, 7524-7525.

86. F. Song, C. Wang, J. M. Falkowski, L. Ma and W. Lin, J. Am. Chem. Soc, 2010, 132, 15390-15398.

87. K. S. Jeong, Y. B. Go, S. M. Shin, S. J. Lee, J. Kim, O. M. Yaghi and N. Jeong, Chem. Sci, 2011, 10.1039/C1030SC00582G.

88. C. Baleizão, B. Gigante, H. Garcia and A. Corma, J. Catal, 2003, 215, 199-207.

89. Y. Gao, J. M. Klunder, R. M. Hanson, H. Masamune, S. Y. Ko and K. B. Sharpless, J. Am. Chem. Soc, 1987, 109, 5765-5780.

90. T. Katsuki and V. Martin, Org. React, 1996, 48, 1-299.

91. S. H. Cho, B. Ma, S. T. Nguyen, H. T.; and T. E. Albrecht-Schmitt, Chem. Commun., 2006, 2563-2565. 